\title{
Early Intervention Protocols: Proposing a Default Bimodal Bilingual Approach for Deaf Children
}

\author{
M. Diane Clark ${ }^{1} \cdot$ Katrina R. Cue $^{1}$ (D) Natalie J. Delgado ${ }^{1} \cdot$ Ashley N. Greene-Woods $^{1} \cdot$ Ju-Lee A. Wolsey ${ }^{2}$
}

Accepted: 29 August 2020 / Published online: 8 September 2020

(c) Springer Science+Business Media, LLC, part of Springer Nature 2020

\begin{abstract}
Despite advances in hearing technology, a growing body of research, as well as early intervention protocols, deaf children largely fail to meet age-based language milestones. This gap in language acquisition points to the inconsistencies that exist between research and practice. Current research suggests that bimodal bilingual early interventions at deaf identification provide children language foundations that can lead to more effective outcomes. Recommendations that support implementing bimodal bilingualism at deaf identification include early intervention protocols, language foundations, and the development of appropriate bimodal bilingual environments. All recommendations serve as multifaceted tools in a deaf child's repertoire as language and modality preferences develop and solidify. This versatile approach allows for children to determine their own language and communication preferences.
\end{abstract}

Keywords Language acquisition · Deaf children · Early intervention $\cdot$ Bimodal bilingual $\cdot$ American Sign Language/ASL

\section{Significance Statement}

Professionals working in the field of Deaf ${ }^{1}$ education ${ }^{2}$ remain stymied by persistent delays in language acquisition persisting across decades despite advances in assistive hearing technology, newborn screenings, early intervention services, research, and knowledge. It would seem that current systematic practices are not adequately addressing persistent language delay issues in the Deaf education field. Therefore, bimodal bilingual interventions are recommended.

Katrina R. Cue

katie.cue@gmail.com

M. Diane Clark

diane.clark@lamar.edu

Natalie J. Delgado

nataliedelgadograce@gmail.com

Ashley N. Greene-Woods

agreene7@lamar.edu

Ju-Lee A. Wolsey

jwolsey@towson.edu

1 Department of Deaf Studies and Deaf Education, Lamar University, 4400 MLK Blvd., P. O. Box 10113, Beaumont, TX 77710, USA

2 Deaf Studies, Interdisciplinary Programs, Towson University, 8000 York Road, Towson, MD 21252, USA

\section{Purpose}

Current practices in the field are typically heavily influenced by the medical model of disability, which focuses on "fixing" the disability in order to allow deaf children to assimilate into the hearing world to the fullest extent possible (Harmon 2013; Valente et al. 2002). As such, a deaf child's early years are often spent honing their listening and speaking skills at the cost of full access to language (Greene-Woods 2020; Hall et al. 2019). Such actions assume that parents must limit their exploration of communication approaches and commit to only one path forward toward language acquisition.

Without early exposure to a fully accessible language, which for deaf children tends to be a visual language (e.g., American Sign Language [ASL]), many children are at risk for language deprivation (Hall et al. 2019) and consequently experience symptoms of language deprivation syndrome as an adult (Hall et al. 2017). Therefore, new research-based policy considerations, deaf adult expertise, and recommendations for changes in existing systems are necessary to support the default practice of bimodal bilingualism from day

\footnotetext{
1 The D/deaf naming conventions outlined by Pudans-Smith et al. (2019) will be adhered to in this manuscript.

2 This article generally focuses on legislation, systems and Deaf education practices in the United States. However, the information we discuss is applicable anywhere.
} 
one of a deaf child's identification to provide the best possible educational outcomes ${ }^{3}$ (Swanwick 2016).

\section{Background}

The Early Hearing Detection and Intervention (EHDI) system has three principal components designed to, "maximize language and communication competence, literacy development, and psychosocial well-being for children who are deaf or hard of hearing" (Joint Committee on Infant Hearing $[\mathrm{JCIH}] 2019$, p. 3). These components or phases are designed to ensure infants are identified as early as possible in order to implement protocols and services with the goal of ensuring developmentally appropriate outcomes related to language and communication (JCIH 2019; National Center for Hearing Assessment and Management [NCHAM] 2019).

\section{EHDI: Hearing Screening}

Historically, the challenges and delays experienced by deaf children came from being identified late leading to missed opportunities for early intervention services to help with language acquisition (Hall et al. 2019). To address this issue, states began implementing mandatory newborn screening. As of 2019, 43 states have some form of legislation regarding Universal Newborn Hearing Screening (NCHAM 2019). However, the term 'universal' is a bit of a misnomer as only 28 of these 43 states require every newborn to be screened, and only 29 out of the 43 states are required to report newborn screening results to their state's Department of Health (NCHAM 2019). Although numbers would indicate that the majority of babies seem to be screened, this statistical majority comes from a small group of states.

\section{EHDI: Identification}

Screening of newborns' hearing levels result in one of two options; pass (no identified hearing differences) or refer with explanation ${ }^{4}$ (a failed hearing screening resulting in a referral to an audiologist for further testing and potential identification). In 2017, the prevalence was 1.7 identified babies for every 1000 babies screened (Centers for Disease Control and Prevention [CDC] 2017). Families that do not follow-up on referrals, receive services, and/or appear in EHDI tracking systems are labeled as loss to follow-up and/

\footnotetext{
${ }^{3}$ Here, we use the sociocultural term of "identification" rather than the medical term of "diagnosis" (Crace and Rems-Smario 2017).

${ }^{4}$ Here, we use the sociocultural term of "refer with explanation" rather than the medical term of "failed hearing test" (Crace and Rems-Smario 2017).
}

or loss of documentation (LFU/LTD). In 2017, $18.4 \%$ of all families screened in the United States and its territories were labeled as LFU/LTD (CDC 2017). These infants and their families need to become the focus on additional tracking for identification to increase positive child outcomes. Then after identification, the next step is enrolling the family and deaf child in early intervention services.

\section{EHDI: Early Intervention}

In 2017 , it was reported that only $65.1 \%$ of identified children were enrolled in early intervention services (CDC 2017). This lack of enrollment compounded with the $18.4 \%$ of LFU/LTD children (CDC 2017) is a sizable gap in the EHDI system with ripple effects typically manifesting in $\mathrm{K}-12$. The EHDI system is only successful if families and deaf children are able to locate, access, and utilize its services to the fullest extent possible.

Early intervention services are additionally important because $96 \%$ of deaf children are born to hearing parents (Mitchell and Karchmer 2004), who frequently have not met a deaf individual prior to their child's identification. The goal(s) during early intervention typically center around speech, hearing, and/or language development (NCHAM 2019). Instead, we propose that optimal early intervention protocols should use a social model approach to disability in which the child is not seen as something to be 'fixed.' Protocols should utilize a strengths-based approach.

\section{The Illusion of Neutrality}

In theory, EHDI for deaf children is believed to remain neutral as far as providing balanced options with all communication approaches (Global Coalition of Parents of Deaf/Hard of Hearing Children [GCPDHHC] 2010). In reality, with the multiple communication approaches and the epistemology of professionals making recommendations, bias influences the system. The continued observation of gaps in language acquisition points to the inconsistencies that exist between research and practice. In practice, EHDI systems tend to largely focus on the listening and speaking modalities for language development (Greene-Woods 2020; Hamilton and Clark 2020). This practice, rather than supporting bilingualism, does not encourage including sign language due to the belief that it is detrimental to listening and speaking skills (Harmon 2013). The GCPDHHC (2010) position statement shows only " $50.9 \%$ of families were given "complete and balanced information about all communication options" (p. 5). This data shows that there is no true balance as the field tends to align with the generally-dominant communication approach preferences, which simultaneously encourages spoken language acquisition and discourages sign language acquisition (Delgado 2020; Hall et al. 2019). 


\section{Bimodal Bilingualism}

The use of sign language at the earliest suggestion of the possibility of being deaf is an efficacious choice for all families, regardless of their preference for modality, as research shows that the use of sign language in infancy stimulates and supports later acquisition of spoken language due to its visual nature being fully accessible to deaf infants (Hassanzadeh 2012; Humphries et al. 2012). Establishing an accessible and comprehensible language as early as possible is essential for neurological and later academic development (Mayberry et al. 2011; Pénicaud et al. 2013). As the delay in acquisition of language increases, the child's neurological development is altered with observed lifelong effects on psycholinguistic processing (Mayberry et al. 2011).

Due to differences in hearing levels and the varying effectiveness of hearing technology, spoken language cannot always be assumed to be fully accessible to deaf children. First, deaf children are often considered to be born with a delay in listening age as hearing fetuses begin auditory learning in the prenatal period (DeCasper and Spence 1986). Then, early amplification at identification allows some children to be able to receive auditory access via traditional amplification methods (e.g., hearing aids), while others get access later through cochlear implants. However, the quality of access to sound is frequently not able to be determined until the child is able to respond using spoken language (e.g., speech discrimination), at which point the child's language has possibly become severely delayed (Greene-Woods 2020). This inability to predict who will benefit from hearing technologies while solely relying on spoken language leads to a large variability in language proficiency (GreeneWoods 2020; Pisoni et al. 2008). In contrast, recent research demonstrates that bimodal bilingual strategies largely avoid these language delays, develop more effective spoken language, as well as provide more effective language and cognitive outcomes than do spoken language interventions alone (Davidson et al. 2014; Hassanzadeh 2012).

\section{Recommendations}

Below, we proffer recommendations, or principles, that support (1) bimodal bilingual early intervention protocols, (2) the development of strong bimodal bilingual language foundations, and (3) align bimodal bilingual research to practices in the P-12 Deaf education system.

\section{Principle \#1: Reframing EHDI}

Effective implementation of a bimodal bilingual philosophy begins at identification. An environment that allows for deaf children to become bimodal bilinguals is effective in avoiding language delays (Hamilton and Clark 2020; Hassanzadeh
2012) and language deprivation (Hall et al. 2017). This foundation for linguistic, cognitive, and academic successes appears to be access to ASL/English bimodal bilingual environments from the point of early deaf identification to at least two years of age (Henner et al. 2016; Hrastinski and Wilbur 2016). The benefits of bimodal bilingualism go beyond having access to both English and ASL; it also serves as a vehicle to scaffold and incorporate the home languages of families who do not speak English (Delgado 2020). Furthermore, it supports the development of a healthy deaf identity, a home cultural identity, and situates the deaf child comfortably between both the Deaf and hearing worlds (Cue 2020; Delgado 2020). In such a scenario, both spoken and signed languages are supported until the child is old enough to demonstrate their own effective language profile.

Protocols for EHDI team visits should include deaf professionals (e.g., Deaf Mentors) in order to introduce families to successful deaf adults utilizing a variety of communication approaches (Hamilton and Clark 2020). Being inclusive of all language opportunities serves to alleviate families' adjustment processes as well as introduce them to a broad range of possibilities for their deaf infant.

Early stages of the EHDI process should demonstrate a singular focus on supporting language opportunities rather than communication options (e.g., modalities and hearing technologies; Hall et al. 2019). At all stages of the EHDI system, the team should be unified in presenting information stressing the effectiveness of bimodal bilingualism. Furthermore, EHDI agencies should welcome partnerships with national Deaf agencies such as the National Association of the Deaf and the American Society for Deaf Children in working together to provide accessible information to families.

\section{Principle \#2: Focus on Language, Not Modality}

We recommend families begin to learn ASL when their child is identified as deaf in order to immediately begin providing sign language exposure. To support that recommendation, family-friendly ASL curriculums needs to be made available and accessible to help hearing, non-signing parents navigate visual and joint attention with their infants. The above practices allow the child to have increased access to information, the environment at large, and to develop close bonds with their family.

Presenting families with timely information about the impact of bimodal bilingual research from the beginning can alleviate the common fear that their child may not be able to communicate with them and share their culture. As a result, deaf children who are bimodal bilinguals are given the agency to make decisions regarding their own communication profile (e.g., keeping, dropping, or continuing to develop their selected modalities and languages) typically 
around two years of age when toddlers have more control of complex linguistic structures.

Deaf children's language acquisition, both signed and spoken/written, should be monitored in a way that just-intime ${ }^{5}$ language interventions can be implemented. The Deaf Mentors, who would be part of the EHDI team, would function to provide language modeling and serve as an oftenoverlooked, yet important, source of support for families of deaf children (Hamilton and Clark 2020). Presenting a unified front as a diversified bimodal bilingual EHDI team will both validate and support parents in providing crucial language opportunities to their deaf infants. These opportunities will also establish the neurological basis for language acquisition and predict success in attaining developmentally appropriate milestones (Hall et al. 2019).

\section{Principle \#3: Continuity of Bimodal Bilingual Practices in K-12 Education}

Early implementation of bimodal bilingual strategies should be continued into K-12 education for continued full language access and academic success. First, the interpretation of a least-restrictive environment and best educational opportunities for deaf children should be reconsidered as current legislation allows for multiple interpretations. Our recommendation is that having direct access to highly qualified teachers specifically trained in Deaf education is essential and translates to greater educational outcomes for deaf children (Chen Pichler et al. 2014). These professionals should be trained in bilingual bimodal educational philosophy and strategies for implementation in the classroom.

Initial research on Deaf schools, ${ }^{6,78}$ and programs utilizing a bimodal bilingual philosophy suggests positive outcomes although examples are limited (Hrastinski and Wilbur 2016; Chen Pichler et al. 2014). One such example is the Parent Infant Program at Kendall Demonostration Elementary School (KDES), which works with families to develop both sign and spoken languages as separate languages and modalities. This bimodal bilingual philosophy is continued in the KDES program, using the bilingual strategy of

\footnotetext{
5 Just-in-time interventions aim to provide the right type and dose of intervention at the right time. Here the intervention would be language interventions when language milestones are delayed (JCIH 2019).

${ }^{6}$ Another exemplar program is at the Rocky Mountain Deaf School, a charter Deaf school in Colorado, which also uses this bimodal bilingual philosophy and creates strong home-school connections (www. rmds.co).

${ }^{7}$ New Mexico School for the Deaf has a strong family program (https://www.nmsd.k12.nm.us).

8 Texas School for the Deaf utilizes a bilingual philosophy (https:// www.tsd.state.tx.us).
}

language separation ${ }^{9}$ to develop strong foundations in both ASL and English. Greater opportunities are needed for deaf and hard of hearing students to develop bilingual bimodal skills to master both languages and modalities in the classroom and beyond. It is recommended that school settings review current practices to assess their alignment with the bilingual bimodal philosophy to boost outcomes.

\section{Discussion}

Above, we point out how decades of neutrality and politics in Deaf education plus the either/or options have served to harm rather than assist the deaf child (Humphries et al. 2012). Consequently, we have highlighted a case for the principles of language foundation that is best viewed as a modified early intervention protocol that incorporates a bimodal bilingual educational philosophy. By giving the deaf child a proverbial toolbox full of tools, they are then empowered to make decisions about their modality and language usage depending upon specific contexts in life (Swanwick 2016). Currently, these decisions are made at identification by EHDI teams that are often heavily biased toward listening and spoken language interventions and unrepresentative of all the professionals needed for effective outcomes (Hamilton and Clark 2020). Language and modality decisions are frequently made with one-sided input, which often leads to language delays and subsequent language deprivation (Hall et al. 2017). We believe that this article could lead to a better understanding of the comprehensive approach that is required for working with deaf children and their families. Within this system is an urgent need for the invaluable expertise of deaf professionals (Crace and Rems-Smario 2017; Hamilton and Clark 2020). A systematic change is needed in transitioning from thinking of deaf children as possessing a deficit, such as having a 'hearing loss,' to thinking of them as simply 'different' (Crace and Rems-Smario 2017).

We must recognize and acknowledge the current era of living through a COVID-19 pandemic, great social and political unrest, frank discussions of racism, and a call for anti-racist education as the way forward for a better society. It has resulted in a reframing of this article as a call to reconsider, reframe, and shift the EHDI system. It is time for the EHDI system to acknowledge that research does not support exclusive listening and speaking practices. For years, deaf adults and their allies have been painted as angry or

\footnotetext{
9 Language separation recognizes the fact that simultaneous communication or sim-com is not $100 \%$ accessible or functional for both languages. As a result, only one language is used at a time for maximum results and access.
} 
radical in protesting instances of oralism, audism, linguicism, and general bias. If we may be ever so brave to say, those deaf adults and their allies are not entirely wrong. In their haste to proceed forward with their knowledge and expertise in prescribing 'best practices,' EHDI professionals have largely neglected the fact that the true expertise lies in the deaf communities. In our discussion, we proffer a final recommendation and that is the fact that each EHDI provider needs to engage in self-reflection and awareness of their own biases and epistemology. In conclusion, the evidence base for bimodal bilingualism is growing, and families and EHDI professionals are finding that sign language does in fact support spoken language (Davidson et al. 2014; Hamilton and Clark 2020; Hassanzedeh 2012; Humphries et al. 2012).

Acknowledgements The genesis behind this paper came from Dr. Clark's work on a task force assembled in Fall 2017 by the Ministry of Education in Ontario, Canada to investigate best practices in Deaf education. From the white paper that was created and circulated informally among professionals, interest began to build. From there, the authors quickly realized that a succinct treatise on the topic with a definite stance that was research-based was much needed within the profession. Thus, the authors decided to expand upon the white paper and create a manuscript for peer-review and publication. This manuscript was born out of the collective knowledge and expertise of the authors and all who they have collaborated with: the Deaf community, professionals, scholars, students, colleagues, and mentors. The people in the field may not always agree regarding how to best approach things; however, we all share the singular goal of the best possible outcome for the deaf child.

\section{Funding None.}

\section{Compliance with Ethical Standards}

Conflict of interest The authors declare that the study was conducted in the absence of any commercial or financial relationships that could be construed as a potential conflict of interest.

\section{References}

Centers for Disease Control and Prevention. (2017). Data and statistics about hearing loss in children. Atlanta, GA: Centers for Disease Control and Prevention.

Chen Pichler, D., Lee, J., \& Lillo-Martin, D. (2014). Language development in ASL-English bimodal bilinguals. In D. Quinto-Pozos (Ed.), Multilingual aspects of signed language communication and disorder (pp. 235-260). Bristol: Multilingual Matters.

Crace, J., \& Rems-Smario, J. (2017). Deaf professionals and community involvement with early education. In National Center for Hearing Assessment and Management (Ed.), eBook: Chapter 15. Retrieved August 14, 2020 from https://www.infanthearing.org/ ehdi-ebook/2017_ebook/15\%2520Chapter15DeafProfessionals 2017.pdf

Cue, K. R. (2020). Hegemonic deaf and hearing cultures of the United States: A deaf ecological systems perspective [Unpublished doctoral dissertation]. Lamar University.

Davidson, K., Lillo-Martin, D., \& Chen-Pichler, D. (2014). Spoken English language development among native signing children with cochlear implants. Journal of Deaf Studies and Deaf Education, 9(2), 238-250. https://doi.org/10.1093/deafed/ent045.

DeCasper, A. J., \& Spence, M. J. (1986). Prenatal maternal speech influences newborns' perception of speech sounds. Infant Behavior and Development, 9(2), 133-150. https://doi. org/10.1016/0163-6383(86)90025-1.

Delgado, N. J. (2020). Mis aspiraciones para mis hijos: Latina immigrant mothers' experiences of early intervention with their deaf children [Unpublished doctoral dissertation]. Lamar University.

Global Coalition of Parents of Deaf/Hard of Hearing Children. (2010). Position statement and recommendations for family support in the development of Newborn Hearing Screening systems (NHS)/Early Hearing Detection and Intervention Systems (EHDI) worldwide. Retrieved August 14, 2020 fromhttps ://www.handsandvoices.org/resources/whitePapers/GPOD.pdf.

Greene-Woods, A. N. (2020). Is spoken language truly accessible?: An investigation on the spoken development in deaf and hard of hearing children [Unpublished doctoral dissertation]. Lamar University.

Hall, M. L., Hall, W. C., \& Caselli, N. K. (2019). Deaf children need language, not (just) speech. First Language, 39(4), 367-395. https://doi.org/10.1177/0142723719834102.

Hall, W. C., Levin, L. L., \& Anderson, M. L. (2017). Language deprivation syndrome: A possible neurodevelopmental disorder with sociocultural origins. Social Psychiatry and Psychiatric Epidemiology, 52(6), 761-776. https://doi.org/10.1007/s0012 7-017-1351-7.

Hamilton, B. M., \& Clark, M. D. (2020). The Deaf Mentor program: Benefits to families. Psychology, 11(5), 713-736. https://doi. org/10.4236/psych.2020.115049.

Harmon, K. (2013). Growing up to become hearing: Dreams of 'passing' in oral deaf education. In J. Brune \& D. Wilson (Eds.), Disability and passing: Blurring the lines of identity (pp. 167198). Philadelphia, PA: Temple University Press.

Hassanzadeh, S. (2012). Outcomes of cochlear implantation in deaf children of deaf parents: Comparative study. Journal of Laryngology and Otology, 126(10), 989-994. https://doi.org/10.1017/ S0022215112001909.

Henner, J., Caldwell-Harris, C. L., Novogrodsky, R., \& Hoffmeister, R. (2016). American Sign Language syntax and analogical reasoning skills are influenced by early acquisition and age of entry to signing schools for the Deaf. Frontiers in Psychology, 7, 1982. https://doi.org/10.3389/fpsyg.2016.01982.

Hrastinski, I., \& Wilbur, R. B. (2016). Academic achievement of deaf and hard of hearing students in an ASL/English bilingual program. Journal of Deaf Studies and Deaf Education, 21(2), 156-170. https://doi.org/10.1093/deafed/env072.

Humphries, T., Kushalnagar, P., Mathur, G., Napoli, D. J., Padden, C., Rathmann, C., et al. (2012). Language acquisition for deaf children: Reducing the harms of zero tolerance to the use of alternative approaches. Harm Reduction Journal, 9(1), 16. https ://doi.org/10.1186/1477-7517-9-16.

Joint Committee on Infant Hearing. (2019). Year 2019 position statement: Principles and guidelines for early hearing detection and intervention programs. Journal of Early Hearing Detection and Intervention, 4(2), 1-44.

Mayberry, R. I., Chen, J. K., Witcher, P., \& Klein, D. (2011). Age of acquisition effects on the functional organization of language in the adult brain. Brain and Language, 119(1), 16-29. https:// doi.org/10.1016/j.bandl.2011.05.007.

Mitchell, R. E., \& Karchmer, M. (2004). Chasing the mythical ten percent: Parental hearing status of deaf and hard of hearing students in the United States. Sign Language Studies, 4(2), 138-163. https://doi.org/10.1093/deafed/enu028. 
National Center for Hearing Assessment and Management. (2019). EHDI legislation: Overview. Logan: National Center for Hearing Assessment and Management.

Pénicaud, S., Klein, D., Zatorre, R. J., Chen, J. K., Witcher, P., Hyde, K., et al. (2013). Structural brain changes linked to delayed first language acquisition in congenitally deaf individuals. NeuroImage, 66, 42-49. https://doi.org/10.1016/j.neuroimage.2012.09.076.

Pisoni, D. B., Conway, C. M., Kronenberger, W. G., Horn, D. L., Karpicke, J., \& Henning, S. C. (2008). Efficacy and effectiveness of cochlear implants in deaf children. In M. Marschark \& P. C. Hauser (Eds.), Deaf Cognition: Foundations and Outcomes (pp. 52-101). Oxford University Press Inc.

Pudans-Smith, K. K., Cue, K. R., Wolsey, J. A., \& Clark, M. D. (2019). To Deaf or not to deaf: That is the question. Psychology, 10(15), 2091-2114. https://doi.org/10.4236/psych.20190.1015135.
Swanwick, R. A. (2016). Deaf children's bimodal bilingualism and education. Language Teaching, 49(1), 1-34. https://doi.org/10.1017/ S0261444815000348.

Valente, J. M., Bahan, B., \& Bauman, H.-D. L. (2002). Sensory politics and the cochlear implant debates. In R. Paludneviciene \& I. Leigh (Eds.), Cochlear implants: Evolving perspectives (pp. 245-258). Washington, DC: Gallaudet University Press.

Publisher's Note Springer Nature remains neutral with regard to jurisdictional claims in published maps and institutional affiliations. 